\title{
White light oblique incidence reflectometer for measuring absorption and reduced scattering spectra of tissue-like turbid media
}

\author{
Guillermo Marquez and Lihong V. Wang \\ Optical Imaging Laboratory, Biomedical Engineering Program, Texas A\&M University, 233 Zachry Eng. Center \\ M.S. 3120, College Station, Texas 77843-3120 \\ LWang@tamu.edu
}

\begin{abstract}
We designed a quick and inexpensive system for spectral measurements of optical properties including absorption coefficient and reduced scattering coefficient. The system was based on oblique incidence reflectometry.[1] A broad band light source was coupled into an optic fiber to deliver light obliquely to turbid media. Nine detection optic fibers were used to collect the diffuse reflectance as a function of source-detector distance. The relative diffuse reflectance profile was used to deduce the absorption and reduced scattering spectra. The system was able to acquire data in a wavelength range of $256 \mathrm{~nm}$ within a fraction of a second.
\end{abstract}

(c) (C1997 Optical Society of America

OCIS codes: (290.5820) Scattering measurements; (300.6170) Spectra

\section{Reference and links}

1. L.-H. Wang and S. L. Jacques, "Use of a laser beam with an oblique angle of incidence to measure the reduced scattering coefficient of a turbid medium," Appl. Opt. 34, 2362-2366 (1995).

2. S. L. Jacques, eds., Laser-Tissue Interaction VIII, Proc. SPIE 2975 (SPIE Press, Bellingham, WA, 1997).

3. E. Sevick-Muraca and D. Benaron, eds., OSA Trends in Optics and Photonics on Biomedical Optical Spectroscopy and Diagnostics, vol. 3 (Optical Society of America, Washington, DC, 1996).

4. B. Chance and R. R. Alfano, eds., Optical Tomography and Spectroscopy of Tissue: Theory, Instrumentation, Model, and Human Studies II, Proc. SPIE 2979 (SPIE Press, Bellingham, WA, 1997).

5. S.-P. Lin, L.-H. Wang, S. L. Jacques and F. K. Tittel, "Measurement of tissue optical properties by the use of oblique-incidence optical fiber reflectometry," Appl. Opt. 36, 136-143 (1997).

6. S.-P. Lin, L.-H. Wang, S. L. Jacques, and F. K. Tittel, "Measurement of absorption and scattering spectra with oblique incidence reflectometry," in OSA Trends in Optics and Photonics on Biomedical Optical Spectroscopy and Diagnostics, E. Sevick-Muraca and D. Benaron, eds., vol. 3, 44-49 (Optical Society of America, Washington, D.C., 1996).

7. G. Marquez, L.-H. Wang, S.-P. Lin, J. A. Schwartz, and S. L. Thomsen, "Anisotropy in the absorption and scattering spectra of chicken breast tissue," Appl. Opt., in press (1997).

8. L.-H. Wang and S. L. Jacques, "Analysis of diffusion theory and similarity relations," Proc. SPIE 1888, 107-116 (1993).

9. T. J. Farrell, M. S. Patterson, and B. C. Wilson, "A diffusion theory model of spatially resolved, steadystate diffuse reflectance for the non-invasive determination of tissue optical properties in vivo," Med. Phys. 19, 879-888 (1992).

10. W. H. Press, B. P. Flannery, S. A. Teukolsky, and W. T. Veterlin, Numerical Recipes in C, 2nd ed. (Cambridge U. Press, Cambridge, UK, 1992), Section 15.5.

\section{Introduction}

It is important to know the optical properties of tissues to properly dose light delivery in therapeutic procedures such as photocoagulation or photodynamic therapy. Also, the optical properties themselves can potentially provide information to monitor tissue metabolic status or diagnose disease.[2-4] This is because light in the visible and near infrared interacts with the subcellular components of tissue, and changes in optical properties of tissues at particular wavelengths could be indicative of disease. 
Optical properties of phantom tissues and biological tissues have been reported using various methods and techniques. However, it remains a challenge to measure the optical properties quickly, reliably, and relatively inexpensively. This paper presents a description of our new system, white light oblique incidence reflectometer (WLOIR). Wang and Jacques first presented oblique incidence reflectometry as a means of measuring the reduced scattering coefficient of biological tissues at a single wavelength,[1] and Lin et al. have demonstrated that absorption and reduced scattering spectra can be easily obtained with this method and with an accuracy of $10 \%$ and 5\%, respectively. [5,6] We recently investigated the effect of muscle fiber alignment on optical properties in biological tissues.[7] Our new system can collect diffuse reflected light from an irradiated sample automatically in a fraction of a second in a wavelength range of $256 \mathrm{~nm}$. The extraction of optical properties from the collected data depended only on the relative profile of the diffuse reflectance based on oblique incidence reflectometry.

\section{Methods and materials}

\subsection{System description}

The experimental system is drawn schematically in Fig. 1. White light (Oriel, $75 \mathrm{~W}$ Xenon Arc Lamp) was coupled to a fiber optic probe made from black delrin and $600 \mu \mathrm{m}$ diameter, low-OH optical fibers.[6] The source fiber was oriented at a $45^{\circ}$ angle of incidence, and nine collection fibers, arranged in a linear array spanning $2.5 \mathrm{~cm}$, collected the diffuse reflectance. The offset between the source plane and the collection plane was $0.12 \mathrm{~cm}$. The indices of refraction of the optic fibers and the liquid phantom were 1.46 and 1.33, respectively. The angle of refraction in the phantom was calculated to be $50.91^{\circ}$. We delivered approximately $4.6 \mathrm{~mW}$ of white light to a tissue-simulating phantom. Although we had a low power light source, the coupling efficiency was high because of the small arc size and the collimating and focusing optics. Also, to correct for slight variations in the collection efficiency from one detection fiber to the next, a correction factor was calculated for each fiber based on a calibration procedure using phantoms of known optical properties.[5] The collection fibers were coupled to a connecting interface. The output of the connection interface was placed at the object plane of the imaging spectrograph.

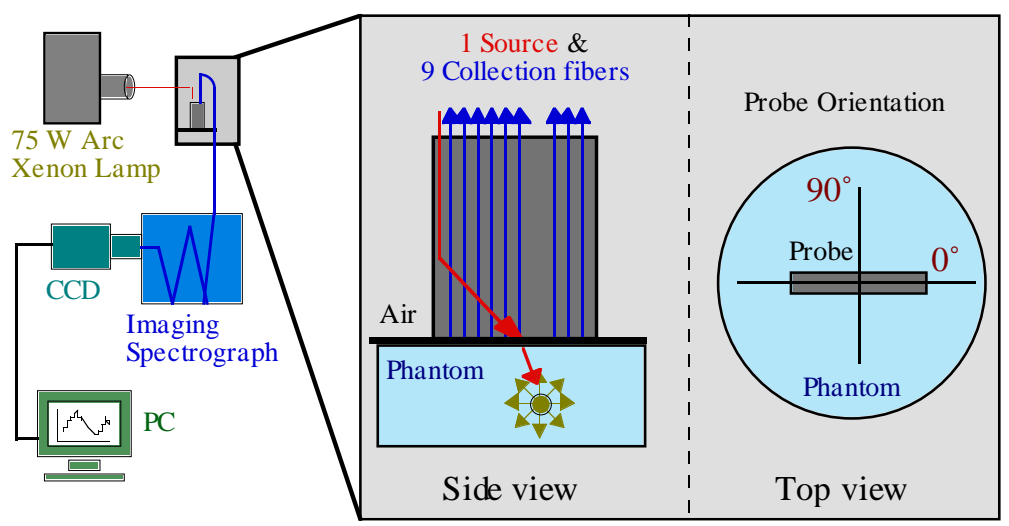

Fig. 1. Schematic of the experimental apparatus. White light was coupled to the obliqueincidence optical fiber probe. A source fiber delivered light to the phantom at an angle of $45^{\circ}$ and the diffuse reflectance was collected by nine collection fibers. All fibers were encased to form a hand-held probe. The collected diffuse reflectance was dispersed by the spectrograph and then imaged onto the CCD matrix. The probe was moved to five different angles to perform five independent measurements.

The spectrograph spectrally dispersed the 1-D light distribution and projected the image onto the CCD matrix. The vertical dimension of the image represented the spatial 
distribution of the diffuse reflectance. The horizontal dimension represented the spectral distribution for light from each collection fiber. The spatial distribution at each wavelength was used to fit for the absorption and reduced scattering coefficients based on the theory to be presented as follows.

The detection system was composed of an imaging spectrograph (Oriel, Multispec 257), a CCD camera (Princeton Instrument Inc., 1530P), and a personal computer to automatically record the spectra of the collected white light. The imaging spectrograph was equipped with toroidal mirrors to minimize astigmatism. The CCD camera had a $512 \times 512$ pixel chip which measured $9.7 \times 9.7 \mathrm{~mm}^{2}$. With this chip size and a 150 lines $/ \mathrm{mm}$ grating (a dispersion angle of $3.2 \mathrm{~nm} / \mathrm{mm}$ ), we were able to image a wavelength range of $256 \mathrm{~nm}$ onto the CCD matrix with spectral resolution of $0.5 \mathrm{~nm}$.

The collected spectra of different collection fibers were found to be misaligned by 1 to $2 \mathrm{~nm}$. We corrected this problem by aligning the characteristic spectral peaks of the collected spectra before the data fitting for optical properties. The peak finding procedure involved finding the characteristic spectral peak of the xenon lamp at $491 \mathrm{~nm}$ for every spectrum. All of the spectra from the collection fibers were aligned such that the characteristic peaks are at the same spectral location. Although the wavelength shift between the collection fibers varied 1 to $2 \mathrm{~nm}$, the extrapolated data showed significant difference before and after the wavelength alignment.

To test the system, we made a liquid phantom composed of absorbing trypan blue dye and $0.9-\mu \mathrm{m}$-diameter scattering polystyrene spheres (PS) with an expected absorption coefficient, $\mu_{\mathrm{a}}$, of $0.26 \mathrm{~cm}^{-1}$ and an expected reduced scattering coefficient, $\mu_{\mathrm{s}}^{\prime}$, of $6.1 \mathrm{~cm}^{-1}$ at $594 \mathrm{~nm}$. The probe was placed on the surface of the phantom. An exposed x-ray film was placed on top of the phantom to approximate a matched boundary condition for diffusion theory. In order to perform independent measurements, the probe was rotated to five different angles with respect to an arbitrary reference point, $0^{\circ}$, and the diffuse reflectance was recorded [Fig. 1]. The absorption and reduced scattering spectra were evaluated for each diffuse reflectance measurement, and the results were averaged. The absorption spectrum of trypan blue dye was measured by collimated transmission. The reduced scattering coefficients of the polystyrene spheres were calculated using Mie Theory at multiple wavelengths.

\subsection{Theoretical}

This section summarizes, for completeness, the theory behind our method described in detail by Lin et al. [5] When light enters a semi-infinite tissue, it will generally scatter a number of times before either being absorbed or escaping the tissue. This scattered light that escapes is called diffuse reflectance. The calculation of absorption coefficient in our method was derived from a diffusion theory-based model of diffuse reflectance although the calculation of the diffusion constant was independent of diffusion theory. [1,5] This diffusion theory model does not accurately model "near diffuse reflectance", i.e., reflectance that falls within the range of 1-2 transport mean free paths $\left(\mathrm{mfp}^{\prime}\right)$ of the source. [8,9] This problem can be avoided by using only the "far diffuse reflectance", i.e., reflectance that falls beyond the range of 1-2 $\mathrm{mfp}$ ' of the source.

For normally incident light, the spatial distribution of diffuse reflectance from a semiinfinite turbid medium has been modeled by two isotropic point sources: one positive source located below the tissue surface and one negative image source above the tissue surface. If light is delivered obliquely, the positive source is buried at the same distance from the point of incidence but with a depth modified by Snell's law. Wang and Jacques found the distance of the buried source to the point of incidence to be most accurately determined by

$$
d_{s}=3 D=\frac{1}{0.35 \mu_{a}+\mu_{s}{ }^{\prime}}
$$


where $D$ is the diffusion coefficient, $\mu_{a}$ the absorption coefficient, and $\mu_{s}{ }^{\prime}$ the reduced scattering coefficient. Therefore, the fundamental difference between normal and oblique incidence is a shift in the positions of the point sources in the $x$ direction [Fig. 2]. This shift, $\Delta x$, is

$$
\Delta x=\frac{\sin \left(\alpha_{t}\right)}{0.35 \mu_{a}+\mu_{s}^{\prime}}
$$

where $\alpha_{t}$ is the angle of refraction, which may be calculated from the relative index of refraction $n$ and the angle of incidence $\alpha_{i}$. The relative index of refraction is unity for a matched boundary.

The modified dipole source diffusion theory model gives diffuse reflectance

$$
R(x)=\frac{1}{4 \pi}\left[\frac{\Delta z\left(1+\mu_{e f f} \rho_{1}\right) \exp \left(-\mu_{e f f} \rho_{1}\right)}{\rho_{1}^{3}}+\frac{\left(\Delta z+2 z_{b}\right)\left(1+\mu_{e f f} \rho_{2}\right) \exp \left(-\mu_{e f f} \rho_{2}\right)}{\rho_{2}^{3}}\right]
$$

where $x$ is the distance between the point of observation and the point of light incidence, $\rho_{l}$ and $\rho_{2}$ are the distances from the two point sources to the point of interest, $z_{b}$ is the distance between the virtual boundary and the surface of the tissue

$$
z_{b}=2 A D
$$

where $A$, a parameter related to the internal reflection is unity for a matched boundary, $\Delta z$ is the depth of the positive point source

$$
\Delta z=\frac{\cos \left(\alpha_{t}\right)}{0.35 \mu_{a}+\mu_{s}^{\prime}}=\Delta x \tan ^{-1}\left(\alpha_{t}\right)
$$

and $\mu_{\text {eff }}$ is the effective attenuation coefficient

$$
\mu_{e f f}=\sqrt{\frac{\mu_{a}}{D}}
$$

Equation (3) can be scaled to fit a relative reflectance profile.

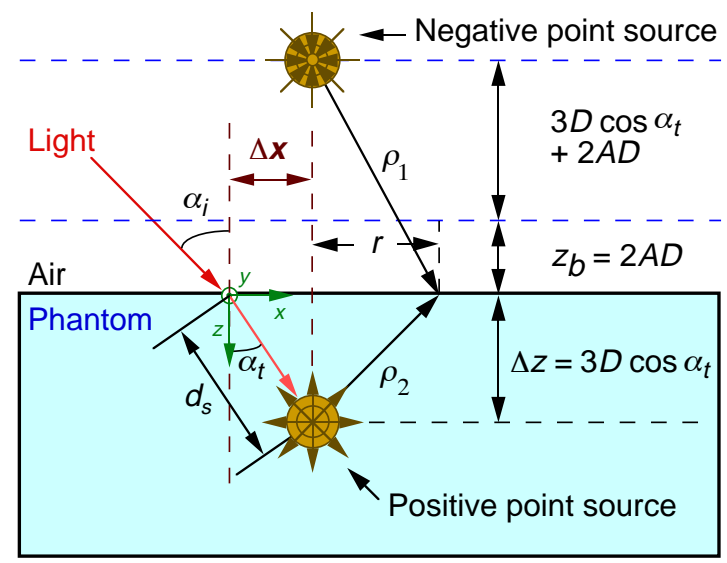

Fig. 2. Schematic representation of obliquely incident light. Note the shift in the center of diffuse reflectance, $\Delta x$. The position of the two point sources in diffusion theory model of oblique incidence reflectometry are shown.

Therefore, the first step in our procedure was to measure the diffuse reflectanceprofile with our fiber optic probe. We used white light for a multi-wavelength measurement. The light collected by each detection fiberwas input to the OMA for spectral resolution. Because we determine the spectrum collected by each fiber, we have in fact measured the spatial distribution of diffuse reflectanceat many wavelengths simultaneously. Thus the reflectance profile at any wavelength can be extracted and analyzed to deduce the optical properties at that wavelength. 
Once the position of the center of far diffuse reflectance relative to the light entry point $(\Delta x)$ was found, the diffusion coefficient was calculated from

$$
D=\frac{\Delta x}{3 \sin \left(\alpha_{t}\right)}
$$

Then a non-linear least squares fit to Eq. (3) with the Levenberg-Marquardt method yielded the effective attenuation coefficient, $\mu_{\text {eff: }}$ [10] The following equations, derived from Eqs. (2), (6) and (7), were used to compute for $\mu_{a}$ and $\mu_{s}{ }^{\prime}$.

$$
\mu_{a}=\frac{\mu_{e f f}^{2} \Delta x}{3 \sin \left(\alpha_{t}\right)}
$$

and

$$
\mu_{s}^{\prime}=\frac{\sin \left(\alpha_{t}\right)}{\Delta x}-0.35 \mu_{a}
$$

\section{Results}

In Fig. 3 we show the effects of wavelength alignment on the extracted optical properties.
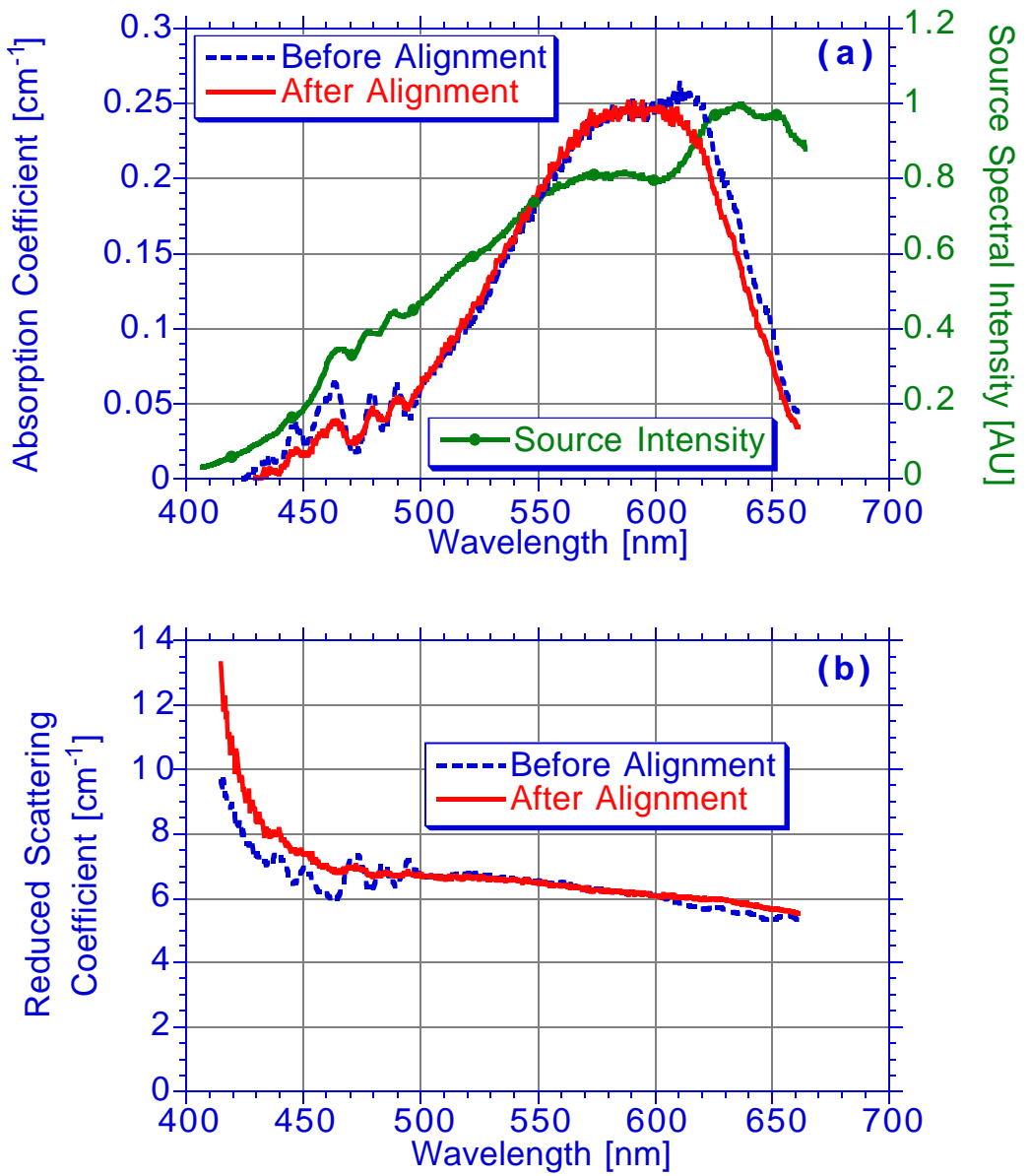

Fig. 3. The extracted optical properties of polystyrene spheres/trypan blue dye phantom before and after the wavelength alignment. (a) The absorption coefficient, $\mu_{a}$, versus wavelength. (b) The reduced scattering coefficient, $\mu_{s}^{\prime}$, versus wavelength. 
Before the wavelength alignment, the artificial spectral peaks in $440-500 \mathrm{~nm}$ in the extracted absorption and reduced scattering spectra were prominent, which introduced significant errors. The artificial peaks were significantly reduced after the wavelength alignment. The source spectrum that was collected by our system shows the characteristic peaks of the xenon lamp at $440-500 \mathrm{~nm}$ [Fig. 3a]. The spectral response of the CCD matrix contributed to the spectral distortion of the source light, which was spectrally flat except for the characteristic peaks. The minor misalignment could be caused by the fiber array in the connecting interface. The collection fibers were not perfectly linear thus caused a small shift in the wavelength between collection fibers as the grating dispersed the input light onto the CCD camera chip.

In Fig. 4a we compared our extracted absorption spectrum with the absorption spectrum of trypan blue dye measured independently using collimated transmission and Beer's law. In Fig. 4b we compared our extracted reduced scattering spectrum to the reduced scattering coefficient of $0.9 \mu \mathrm{m}$ diameter polystyrene spheres calculated using Mie Theory at several wavelengths. Figure 4 shows very good correlation between our extracted optical properties and the expected values except for the short wavelength region. The discrepancy in the short wavelength was caused by the low spectral sensitivity of the CCD matrix evidenced by the measured spectrum of the source in Fig. 3a.
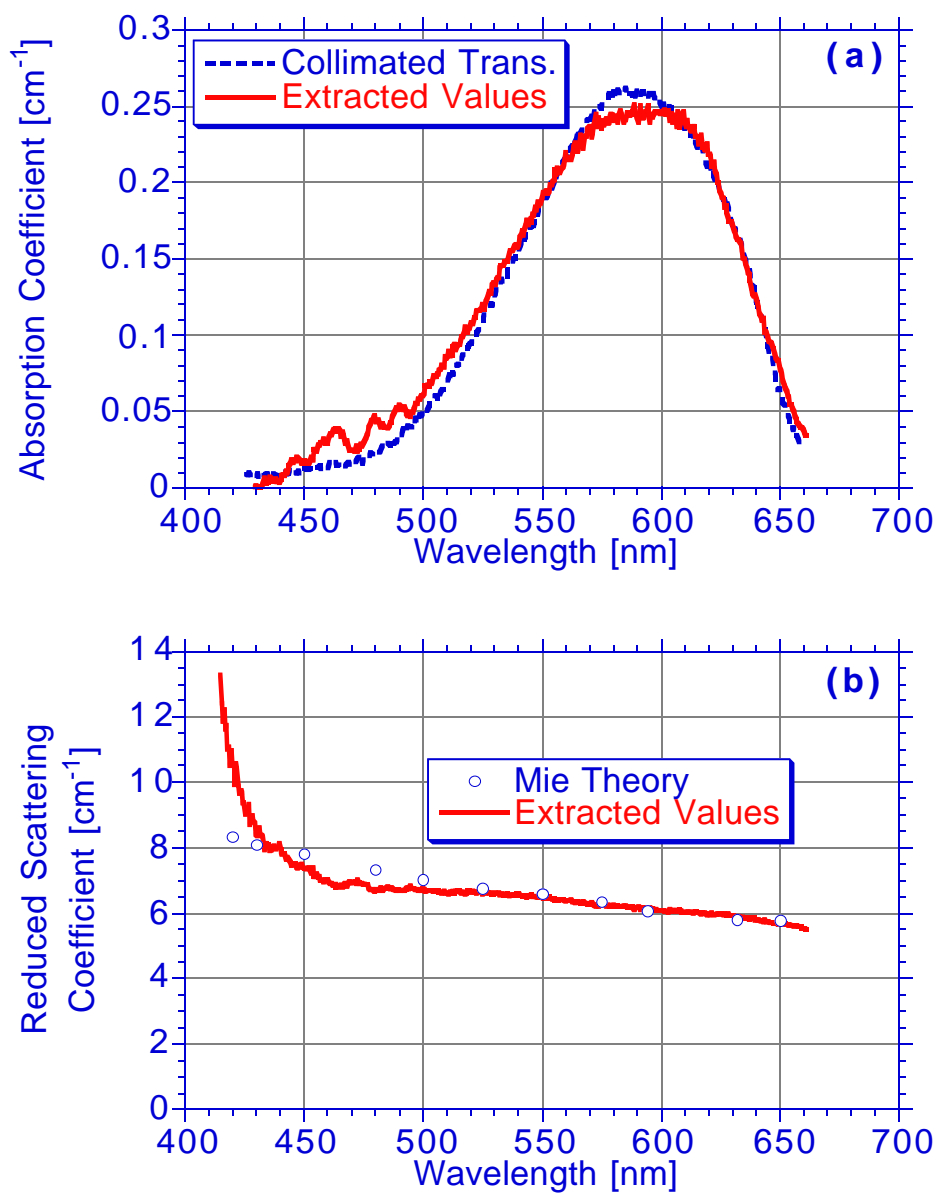

Fig. 4. The average optical properties of polystyrene spheres/trypan blue dye phantom. (a) The absorption coefficient, $\mu_{a}$, versus wavelength. (b) The reduced scattering coefficient, $\mu_{s}^{\prime}$, versus wavelength. 


\section{Conclusion}

This paper presented a new experimental system, white light oblique incidence reflectometer, that can quickly collect the spectra of the diffuse reflected light distribution from an irradiated sample automatically in a fraction of a second for a spectral range of $256 \mathrm{~nm}$ with spectral resolution of $0.5 \mathrm{~nm}$. The collected spectral data can be processed to extract the spectra of absorption and reduced scattering coefficients of turbid media based on oblique incidence reflectometry. Only the relative profile of the diffuse reflectance was used for the extraction of optical properties, which made the device insensitive to variations of some system parameters such as source power. The experimental measurements on the turbid phantom verified our experimental system. This device has potential applications in non-invasive real-time diagnosis and monitoring of superficial lesions in medicine and may find applications in other fields related to turbid media.

\section{Acknowledgments}

Special thanks to Q. Shen, T. Bendele, and N. Thukral for their contributions to this project. The project was sponsored in part by The Whitaker Foundation grant and the National Institutes of Health grants R29 CA68562 and R01 CA71980. 